\title{
Modelling and design of a direct-drive lift control with rope elasticity and estimation of starting torque
}

\author{
S. Bolognani, A. Faggion, L. Sgarbossa \\ Dept. of Electrical Engineering - University of Padova \\ Via Gradenigo 6/A - I - 35131 Padova - Italy \\ bolognani@die.unipd.it
}

\author{
L. Peretti \\ Dept. of Engineering and Management \\ University of Padova - Vicenza, Italy \\ luca.peretti@unipd.it
}

\begin{abstract}
This paper deals with position control of a direct drive rope-based lift system at mechanical brake releasing. The electrical equipment includes a high-torque, low-speed permanent magnet synchronous motor with external rotor directly connected to the rope pulley. The drive uses only stator current sensors for torque control and an incremental encoder on the motor shaft. A detailed model of the system, which comprises also rope elasticity, is presented, as wells as a load torque estimation algorithm and a feedforward action on the torque reference signal. Such reference is used to reduce the cabin falling down at brake releasing below a perceptible threshold. Final experimental results support the mathematical theory outlined in the paper, validating the proposed control strategy.
\end{abstract}

\section{INTRODUCTION}

Rope-based lift systems are widely used in civil and commercial buildings. Modern lift technology moves toward direct drive solutions, where a high-torque low-speed motor is directly connected to the rope pulley without the need of any speed reduction gear box. Such a solution allows the elimination of the gear-motor room, with advantages in terms of volume and costs. Reliability of the whole system is also improved, while the need of maintenance is greatly reduced. Direct drive solutions also stimulates the design of control strategies which are not applicable in conventional configurations. In the case of rope-based lift systems, the main issue should be an accurate and comfortable motion control of the cabin in any working condition, while using a minimal set of sensors and transducers in order to reduce costs and to increase reliability.

This paper deals with the motion control of a rope-based lift system equipped by a direct-drive permanent magnet synchronous motor controlled by a current (torque) loop and an external speed loop. In particular, the paper is devoted to the control of the cabin at the floor level after the door is closed and mechanical brakes are released. Comfort specifications require that any tremor of the cabin floor at the brake releasing has to be as small as possible. To solve the task the paper proposes an effective estimation algorithm of the load torque applied to the motor pulley, which is due to the unbalanced suspended bodies of the elevator system. The torque estimation is performed rapidly just after sending the release command to the brakes, using only the information received by the incremental encoder installed on the motor shaft. A feed-forward action is applied at the output of the PID speed controller to generate an enhanced torque reference with the purpose of obtaining a minimum speed error. Since the speed reference signal is equal to zero, the overall result is a minimized cabin movement. The paper is organized as follows. In Sect. II a detailed dynamic model of the lift system is carried out taking into account also rope elasticity. Sect. III analyses the effects of the load torque at the brake releasing and a load torque estimation algorithm is proposed and illustrated. In Sect. IV the proposed estimation technique is verified on an experimental test bench together with the feedforward action, pointing out the benefits of the proposed control strategy. Conclusive remarks will close the paper.

\section{SySTEM MODELLING}

A schematic of a Direct-Drive (DD) lift system is shown in Fig. 1. The system includes the pulley $\mathrm{P}$ directly connected to the Permanent Magnet (PM) synchronous motor that moves, by means of the ropes, the counterweight $\mathcal{M}_{1}$ and the cabin $\mathcal{M}_{2}$. Presence of rope elasticity is pointed out in the figure. Brakes are applied to the pulley during the stop phase of the system, where angular position $\theta$ is assumed equal to zero. Coordinates $x_{1}$ and $x_{2}$ return the position of counterweight and cabin with respect to their steady-state position at $\theta=0$.

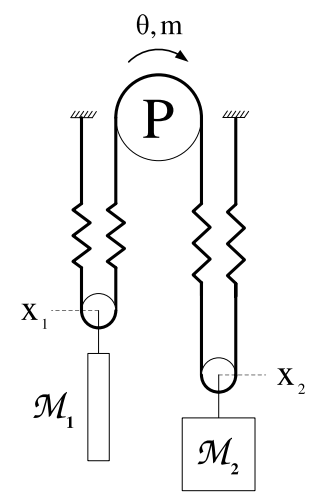

Fig. 1. Schematic model of Direct-Drive lift system

The DD motion control is closed on the speed signal obtained by the speed sensor (encoder) connected to the motor shaft. Motor currents are also available and a high performance 
closed loop vector control has been implemented for the stator currents. No other transducer is installed for the motion control task. In order to design the DD speed loop, the Transfer

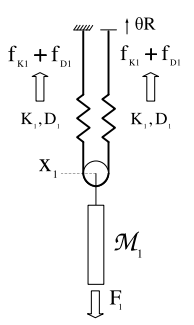

(a) Counterweight

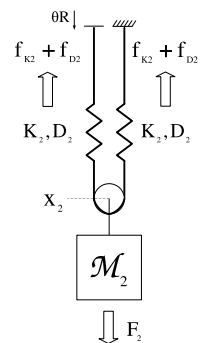

(b) Cabin

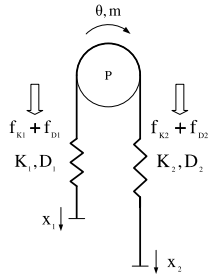

(c) Motor pulley
Fig. 2. Schematic of DD lift sub-system

Function (TF) between motor torque as input and motor speed as output, has to be derived. First of all, for that purpose, dynamic motion equations of the three sub-systems of Fig. 2 are carried out. For sub-system 2(a), applying the force balance, one can obtain (lower letters are for time-dependent variables, capital letters for constant coefficients):

$$
\mathcal{M}_{1} \ddot{x}_{1}=-2 K_{1} x_{1}-K_{1} R \theta-2 D_{1} \dot{x}_{1}-D_{1} R \dot{\theta}
$$

where $K_{1}$ and $D_{1}$ represent the rigidity and the damping coefficient of the rope on the counterweight side, while $\mathrm{R}$ is the pulley radius. Weight force $F_{1}=\mathcal{M}_{1} g$ (g gravity acceleration) of the mass $\mathcal{M}_{1}$ does not appear in (1) as it is balanced by the elastic force $K_{1} x_{10}$, where $x_{10}$ is the steady-state elongation of the ropes.

In a similar way, the cabin (sub-system 2(b)) is described by the following expression:

$$
\mathcal{M}_{2} \ddot{x}_{2}=-2 K_{2} x_{2}+K_{2} R \theta-2 D_{2} \dot{x}_{2}+D_{2} R \dot{\theta}
$$

where $K_{2}$ and $D_{2}$ are the rigidity and the damping coefficient of the rope on the cabin side. Again, weight force $F_{2}=\mathcal{M}_{2} g$ of the mass $\mathcal{M}_{2}$ does not appear in (2) as it is balanced by the elastic force $K_{2} x_{20}$, with $x_{20}$ being the steady state elongation of the ropes.

At last, for sub-system 2(c), the motor-side torque balance returns the following equation:

$$
\begin{aligned}
J_{p} \ddot{\theta} & =m+\left(-\frac{F_{1}}{2}+\frac{F_{2}}{2}\right) R+ \\
& +\left(f_{K 2}-f_{K 1}+f_{D 2}-f_{D 1}\right) R
\end{aligned}
$$

where $\mathrm{m}$ is the motor torque and

$$
\left.\begin{array}{l}
f_{K 1}=K_{1}\left(x_{1}+\frac{\theta}{2} R\right) \\
f_{K 2}=K_{2}\left(x_{2}-\frac{\theta}{2} R\right) \\
f_{D 1}=D_{1}\left(\dot{x}_{1}+\frac{\dot{\theta}}{2} R\right) \\
f_{D 2}=D_{2}\left(\dot{x}_{2}-\frac{\dot{\theta}}{2} R\right)
\end{array}\right\}
$$

are respectively elastic forces and damping forces [1]. From (1), (2) and (3) one can realizes that input of the system is $m_{e q}=m+\left(-F_{1}+F_{2}\right) R / 2$ which is the unbalanced torque on the motor pulley, and the whole mechanical system can be represented by the block diagram of Fig 3 .

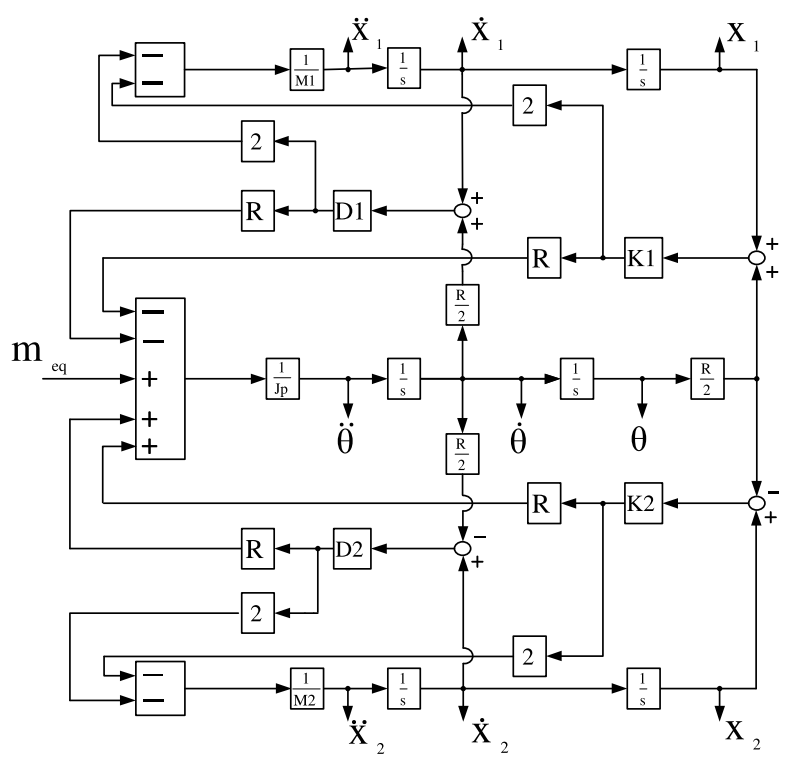

Fig. 3. Block diagram of Direct-Drive lift system

Eqs. (1), (2) and (3) can be transformed into the s-domain, obtaining the following expression:

$$
\begin{aligned}
T F(s) & =\frac{\Omega(s)}{M(s)} \\
& =\frac{\left(s^{2}+s \frac{2 D_{1}}{\mathcal{M}_{1}}+\frac{2 K_{1}}{\mathcal{M}_{1}}\right)\left(s^{2}+s \frac{2 D_{2}}{\mathcal{M}_{2}}+\frac{2 K_{2}}{\mathcal{M}_{2}}\right)}{J_{p} s\left(s^{4}+a_{D 3} s^{3}+a_{D 2} s^{2}+a_{D 1} s+a_{D 0}\right)}
\end{aligned}
$$

where $a_{D 3}, a_{D 2}, a_{D 1}, a_{D 0}$ are reported in Appendix.

Fig. 4 shows the Bode diagram of $T F(s)$ in the case $K_{1}=K_{2}$. From the figure, it can be seen (and proved) that $T F(s)$ exhibits a sequence of second order zeros and poles, in addition to the pole and the zero at null and infinite frequency respectively. On the basis of $\mathrm{TF}(\mathrm{s})$ (4), the speed control loop can be designed [2].

\section{LOAD TORQUE EFFECT AND ESTIMATION}

When the brakes are released, the unbalanced situation that appears on the motor pulley involves a system acceleration whose direction and intensity are unknown, as it depends on the number of people in the cabin. The dynamics can be examined by Fig. 5. Torque estimation $m_{e}$ is a contribution to the PID speed controller output that will be discussed later and can be ignored here. Provided that the brakes are released instantaneously, torque disturbance is a step-shaped torque given by $M_{0}=\left(-F_{1}+F_{2}\right) R / 2$, while the torque given by $f R$ , with $f$ expressed by (3b), is equal to zero in any steady state condition. Speed reference is kept equal to zero to maintain the cabin at the floor level. However, the cabin position does 

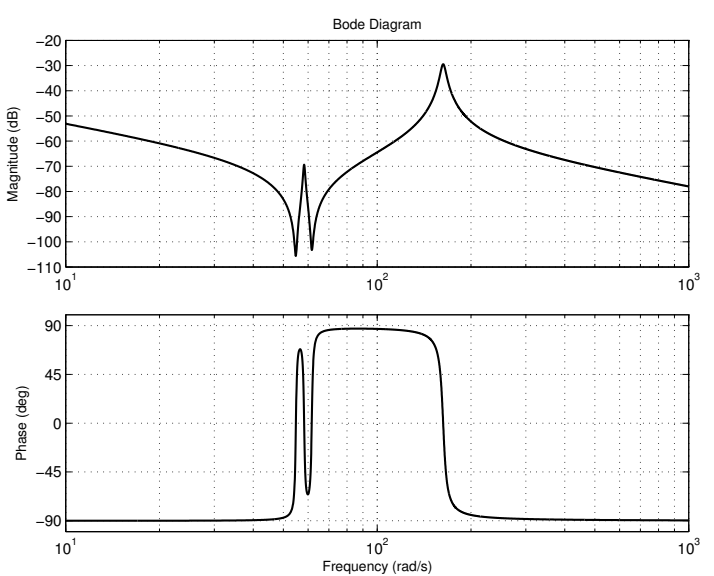

Fig. 4. Bode diagram of $T S(s)$ considering the damping

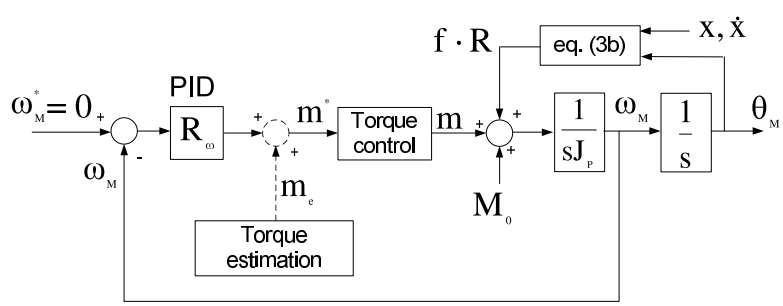

Fig. 5. Motion control schema

not remain equal to zero, due to torque disturbance caused by the unbalanced contribution of cabin and counterweight.

It is worth noticing that steady-state position error at the floor level after the brakes are released and motion is controlled by the speed loop of Fig 5, is proportional to load torque amplitude. This can be proved by

$$
m(\infty)=-M_{0}=K_{I} \int_{0}^{\infty} \omega d t=K_{I} \theta(\infty)
$$

where $K_{I}$ is the integral gain of the PID controller. In writing (5) it has been taken into account that proportional and derivative contributions of the speed controller are null at steady state and it as been assumed, for the sake of simplicity, a unity gain of the torque control.

From (5) one attains

$$
\theta(\infty)=\frac{M_{0}}{K_{I}}
$$

Eq. (6) confirms the well-known elastic behaviour of a PID speed control under null speed reference: angular movement is proportional to the applied load torque.

In order to reduce angular movement and therefore the cabin movement after brake releasing, the integral gain has to be increased as much as possible. However elasticity of the ropes causes an upper limit of the PID speed control bandwidth and consequently an upper limit of the integral gain [2].

An alternative way is represented by the introduction of a feedforward action by adding an estimation of the load torque to the output of the speed controller, avoiding the torque reference to be entirely generated by the integral action of the speed controller. In ideal conditions, if the estimation is exactly coincident to the load torque $M_{0}$ and the torque control has an unity gain and negligible delay, no movements will occur as can be easily recognized from Figs. 3 and 5. Of course, this is not achievable unless another transducer is used in addiction to the encoder and the Hall sensors, or the load torque is estimated. The problem of angular movement reduction is therefore focused on a rapid and effective estimation of the load torque and the subsequent application of such signal as a feedforward contribution.

To this purpose, one can observe that motor torque is initially null and pulley movement is initiated by the load torque $M_{0}$. Initial acceleration of the pulley is therefore given by

$$
\dot{\omega}(0)=\lim _{s \rightarrow \infty} s(s \Omega(s))=\lim _{s \rightarrow \infty} s^{2} T F(s) \frac{M_{0}}{s}=\frac{M_{0}}{J_{p}}
$$

where a load torque given by a step function as been assumed. From (7) it results:

$$
M_{0}=\dot{\omega}(0) J_{p}=\alpha(0) J_{p}
$$

which suggest that the load torque can be estimated through the measurement of the initial motor acceleration $\alpha(0)$, given the motor inertia.

The algorithm used to estimate the torque compare actual motor position $\theta_{k}$ delivered by the encoder at the sampling time $t_{k}$ with the estimated angular position $\tilde{\theta}_{k}$ that is given by the parabolic relationship

$$
\tilde{\theta}_{k}=\frac{1}{2} \alpha t_{k}^{2}
$$

In writing (9), a constant acceleration has been assumed during the short interval of the torque estimation.

The angular acceleration in (9) is evaluated by minimizing the quadratic error of comparison which yields to

$$
\alpha=2 \frac{\sum_{N} \theta_{k} t_{k}^{2}}{\sum_{N} t_{k}^{4}}=\frac{2}{T_{s}^{2}} \frac{\sum_{N} \theta_{k} k^{2}}{\sum_{N} k^{4}}
$$

where the comparison is applied for the first $\mathrm{N}$ samples and $T_{s}$ is the sampling period.

Eq. (10) is applied iteratively, starting from the instant in which the load torque is applied. When a reliable acceleration is attained (denoted by a maximum value carried out by (10)), estimation is stopped and the feed-forward action is launched. Some details on the practical implementation will be given in the next section.

\section{EXPERIMENTAL RESULTS}

In this section, some details on the laboratory test bench and the first experimental results are shown. Experimental verification of the proposed lift motion control has been carried out by using an actual motor drive designed for a lift system, as shown in Fig. 6, [3]. The motor is a SurfaceMounted Permanent Magnet three-phase synchronous motor with external rotor, whose parameters are reported in Tab. 
I, together with the essential information about the control system. In Fig. 6 shows also the brakes that work on a disk which is axially connected to the rotor.

TABLE I

\begin{tabular}{|c|c|c|c|}
\hline \multirow{2}{*}{} & \multicolumn{3}{|c|}{ SPM Motor with external rotor } \\
\cline { 2 - 4 } & Description & Value & Unit \\
\hline 1 & Nominal Voltage $\left(U_{a}\right)$ & 380 & $\mathrm{~V}$ \\
\hline 2 & Nominal Current $\left(I_{n}\right)$ & 24 & $\mathrm{~A}$ \\
\hline 3 & Nominal Torque $\left(M_{n}\right)$ & 320 & $\mathrm{Nm}$ \\
\hline 4 & Pole pairs & 12 & $\mathrm{~m}$ \\
\hline 5 & Pulley radius & 0.17 & Control system \\
\hline & \multicolumn{3}{|c|}{} \\
\hline 6 & Position measure: encoder 2048 ppr with SPI absolute position \\
\hline 7 & \multicolumn{2}{|c|}{ Inverter: three-phase IGBT with 10 kHz PWM } \\
\hline 8 & Controller board: equipped with Freescale DSP 56F803 \\
\hline
\end{tabular}

A spring system have been used to simulate a real lift installation. The right picture of Fig. 6 clearly shows the spring which reproduces the torque effect due to an unbalanced cabin-counterweight combination.

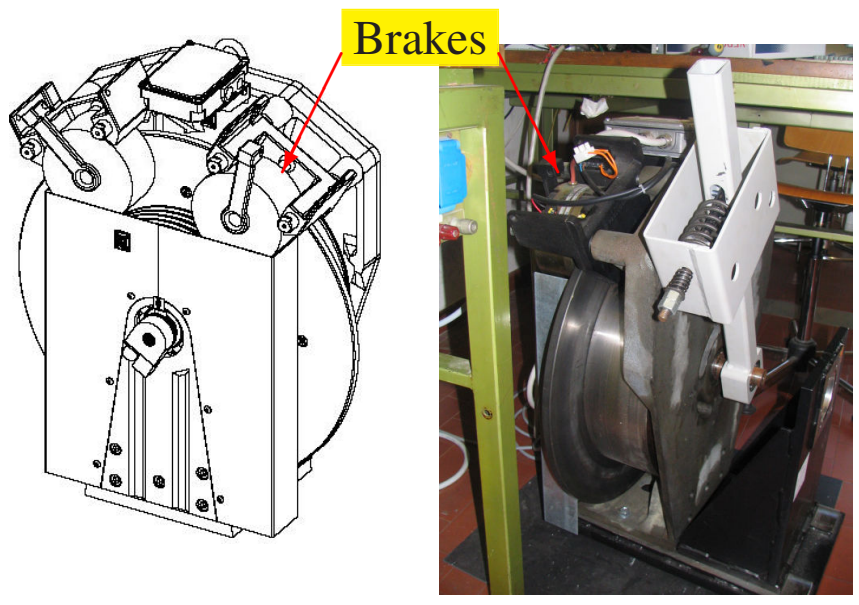

Fig. 6. Motor drawing (left) and mass-spring test bed (right)

During the experimental operation, the time interval between the brakes opening command and the actual brake release (and thus the initial movement of the motor) is unknown because of the uncertain and non repetitive behaviour of the brake system. To overcome this trouble, the time origin for the estimation algorithm start is fixed at the occurrence of the second encoder pulse in one or in the other direction. Then the algorithm starts and, after some milliseconds, the final torque is estimated, much earlier than the integration in the PID controller is completed.

The feedforward action anticipate a substantial part of the steady state reference of the torque which is required to sustain the load. The advantage in term of reduced cabin movement can be clearly recognized in Fig. 8, where measured pulley movement (expressed in encoder pulses) with and without feedforward action are shown. Each pulse corresponds to a cabin movement of about $65 \mu \mathrm{m}$. The advantage of using the load torque estimation and the feedforward contribute is evident as the cabin movement is approximately reduced within $0.5 \mathrm{~mm}$ (instead of more than $1.5 \mathrm{~mm}$ ), which is tolerable by the comfort requirements.

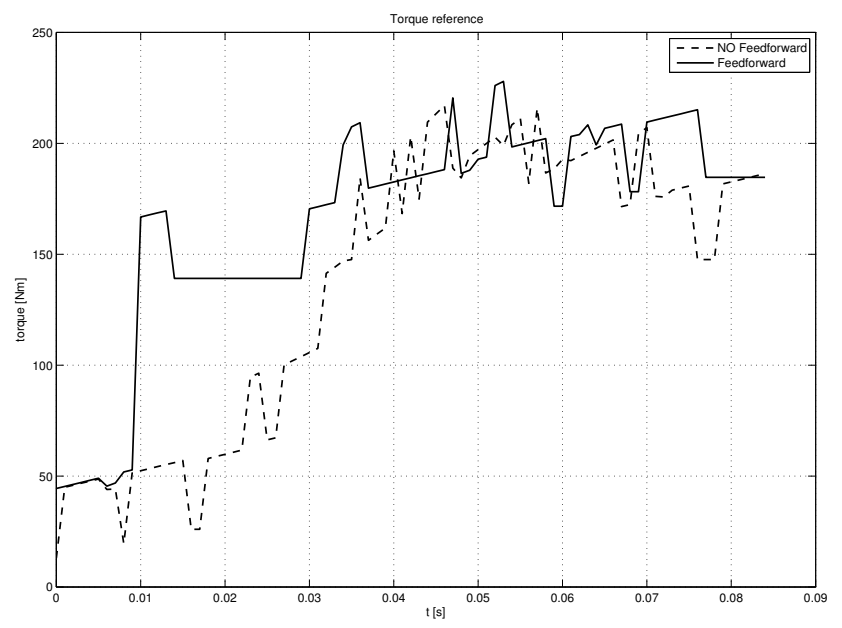

Fig. 7. Torque reference with and without feed-forward action

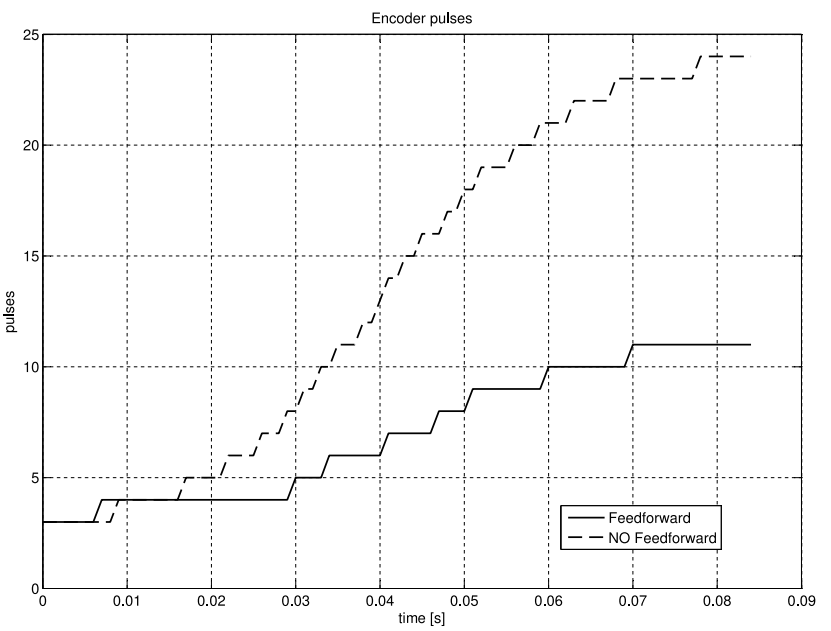

Fig. 8. Motor position after the release of the brakes, with and without feed-forward action

\section{CONCLUSION}

The paper deals with the position control of a direct drive rope-based lift system at the mechanical brake releasing. The electrical equipment include a high-torque, low-speed permanent magnet synchronous motor with external rotor directly connected to the rope pulley. The drive uses only stator current sensors (for torque control) and an incremental encoder on the motor shaft.

An effective estimation algorithm of the load torque applied to the motor pulley, which is caused by the unbalanced suspended bodies of the elevator system, have been presented. The torque estimation is rapidly performed just after the release command is sent to the brakes and is based only on the information received by the incremental encoder installed on the motor 
shaft. A feed-forward action is applied to the output of the PID speed controller to achieve a minimum speed error and then, consequently, a reduced cabin movement. Experimental results have been provide to validate the proposed solution.

\section{ACKNOWLEDGMENT}

The authors wish to thank Company Daldoss srl, Italy, for providing the motor drive equipment

\section{REFERENCES}

[1] Y.Takeichi, S.Komada, M.Ishida, T.Hori, Speed Control of Symmetrical Three-Mass Resonant System by PID-Controllers, Proceedings of IEEE Advanced Motion Control Workshop, pp. 594-599, Mie University, Japan, 1996.

[2] S. Bolognani, A. Venturato, M. Zigliotto, Therotical and Experimental Comparison of Speed Controller for Elastic Two-Mass Systems, Proceedings of IEEE Power Electronics Specialists Conference, CD-ROM, Galway (IRL), 2000.

[3] N.Bianchi, G.Grezzani, G.Ciammetti, External-rotor surface-mounted permanent magnet motor for direct-drive elevator systems, Power Conversion and Intelligent Motion Conference, 7-9 June, CD-ROM, Nuremberg (D), 2005.

\section{APPENDIX}

As indicated in (4), the coefficient in the denominator are given by:

$$
\begin{aligned}
& a_{D 3}= \frac{2 D_{1}}{\mathcal{M}_{1}}\left(1+\frac{R^{2} \mathcal{M}_{1}}{4 J_{p}}\right)+\frac{2 D_{2}}{\mathcal{M}_{2}}\left(1+\frac{R^{2} \mathcal{M}_{2}}{4 J_{p}}\right) \\
& a_{D 2}= \frac{2 K_{1}}{\mathcal{M}_{1}}\left(1+\frac{R^{2} \mathcal{M}_{1}}{4 J_{p}}\right)+\frac{2 K_{2}}{\mathcal{M}_{2}}\left(1+\frac{R^{2} \mathcal{M}_{2}}{4 J_{p}}\right)+ \\
&+\frac{4 D_{1} D_{2}}{\mathcal{M}_{1} \mathcal{M}_{2}}\left(1+\frac{R^{2} \mathcal{M}_{1}}{4 J_{p}}+\frac{R^{2} \mathcal{M}_{2}}{4 J_{p}}\right) \\
& a_{D 1}= \frac{4 D_{1} K_{2}}{\mathcal{M}_{1} \mathcal{M}_{2}}\left(1+\frac{R^{2} \mathcal{M}_{1}}{4 J_{p}}+\frac{R^{2} \mathcal{M}_{2}}{4 J_{p}}\right)+ \\
&+\frac{4 D_{2} K_{1}}{\mathcal{M}_{1} \mathcal{M}_{2}}\left(1+\frac{R^{2} \mathcal{M}_{1}}{4 J_{p}}+\frac{R^{2} \mathcal{M}_{2}}{4 J_{p}}\right) \\
& \frac{4 K_{1} K_{2}}{\mathcal{M}_{1} \mathcal{M}_{2}}\left(1+\frac{R^{2} \mathcal{M}_{1}}{4 J_{p}}+\frac{R^{2} \mathcal{M}_{2}}{4 J_{p}}\right) \\
& a_{D 0}
\end{aligned}
$$

